\title{
Optimizing Analytical Complexities in Emerging Field-Portable Chemical Detection Instruments for Harsh Environments and Public Health Science
}

\author{
Anton Shufutinsky ${ }^{1,2,3,4,5 *}$ and Hildehardo Viado 5,6 \\ ${ }^{1}$ Changineering Global, USA \\ ${ }^{2}$ Academy of Interdisciplinary Health Science Leaders, USA \\ ${ }^{3}$ Nova Southeastern University, USA \\ ${ }^{4}$ Uniformed Services University of the Health Sciences, USA \\ ${ }^{5}$ Department of Industrial Hygiene, Naval Hospital Camp Pendleton, USA \\ ${ }^{6}$ US Army Defense Threat Reduction Agency, USA
}

Submission: January 08, 2019; Published: May 23, 2019

*Corresponding author: Anton Shufutinsky, Changineering Global, Cheltenham, USA

Abstract

Exposure scientists often use gas chromatography-mass spectrometry (GC-MS) systems for analysis of hazardous chemicals [1-3]. Because GC-MS systems with transmission quadrupole mass spectrometers transmission quadrupole mass spectrometers (TQMS) historically have limited portability, small ion trap mass spectrometers (ITMS) have been manufactured to reduce size and increase ruggedness of GC-MS systems [2, 4-8].

The formation of pseudomolecular ions observed in ITMS spectra are seen as a drawback, potentially leading to chemical misidentification [9]. It is proposed here that these spectral complexities can improve analytical power if the pseudomolecular ions can be used to differentiate chemical isomers, not possible with TQMS. Isomers of propyl and butyl sulfide were analyzed using TQMS and ITMS. TQMS spectra exhibited nearly identical peaks for sulfide isomers.

ITMS yielded complex spectra with protonated molecules, dimers, and adducts. Differentiation of sulfide isomers we accomplished via characteristic spectral differences in the pseudomolecular ions formed during detection. The research shows that field-portable ITMS systems can differentiate chemical isomers, a capability that may be important for analysis of toxic industrial chemicals and chemical warfare agents.

Keywords: Gas Chromatography; Mass Spectrometry; Harsh Environments; Public Health Chemistry

\section{Introduction}

In the exposure sciences, a need exists for the development of high-performance analytical equipment with increased portability. One way in which manufacturers are continuously working to meet that need is through advancement of emerging technologies to improve GC-MS chemical identification instrumentation, making detection systems smaller and faster for field use while striving to maintain or improve equipment analytical performance. One such advancement is the advent of field-portable (FP) GC-MS instruments that have been and continue to be used in a variety of settings over the past two decades.

Although there are a number of GC-MS systems that are considered portable, they vary in size, weight, power requirements, attachment needs, sample introduction capabilities, and ease of use. Some field portable GC-MS (FP-GC-MS) systems are vehi cle-mounted or field-portable but stationary, while others are truly person-portable, capable of solitary movement and operation in theoretically every environment in the field by one or two individuals.

The overall purpose of this study was to assess the instruments that have emerged as field portable and capable and evaluate the previously reported chemical reactions that occur in ITMS systems vs TQMS for analytical capability and optimization rather than potential problems and weaknesses, with the overall goal being to optimize methods for analysis of hazardous compounds in deployed, emergency management, forensic investigation, and public health preparedness settings. This was achieved through the evaluation of field-portable GC-MS units for the analysis of common industrial hazardous compounds with partial similar 
structures to hazardous compounds and chemical warfare agents (CWA) or their components. For legal, logistical, and safety purposes, live chemical warfare agents could not be used for this study.

\section{Hypothesis}

Ion/molecule reactions that occur during chemical analysis using small internal ionization ion trap mass spectrometers (ITMS) will result in the formation of pseudomolecular ions, and these psuedomolecular ion byproducts can be used to differentiate isomers of military and emergency-management relevant chemicals.

\section{Background}

\section{Emergency operations and accidental hazardous mate- rials releases}

Military, forensic, and emergency response operations are often unique when compared to the day-to-day operations in the civilian industrial complex, often using unique equipment and processes, and occurring in unusual environments in which short notice, on-the-spot solutions to unexpected operational requirements are not unheard of. The heavy industrial, unexpected, and high-hazard culture of the military lends itself to the potential for unexpected emergency responses to accidental chemical releases and unanticipated exposures to toxic industrial chemicals [10] as do first response organizations and forensic investigators.

Over the past two decades, there has been a noticeable increase in the need to establish effective exposure assessment programs in harsh environments, including military industrial processes, deployed settings, emergency response and public health preparedness programs [10-12], and chemical, biological, radiological, nuclear, and high-yield explosive (CBRNE) programs. The ability to effectively analyze environmental matrices within these settings can be vital in decision-making regarding forward deployment of military troops, law enforcement agents, or emergency responders, as well as decisions regarding post-exposure medical and environmental responses, and selection of personal protective equipment, among other necessary actions. These situations increase the need for rapid field-based exposure monitoring equipment.

The existence of field-deployed high-performance analytical equipment can potentially provide the same level of analytical results as a laboratory, but in a matter of minutes, providing deployed commanders the capability to make near real-time decisions when they are necessary. Rapid on-site field-based chemical identification can also improve decision-making that will ensure increased safety and protection of troops and responders in high-hazard environments [13]. Gas chromatography-mass spectrometry (GC-MS) is a technology that has proven to be well-suited for identification of dangerous organic chemicals, including toxic industrial compounds (TIC), toxic industrial materials (TIM), and chemical warfare agents (CWA).
The coupling of GC and MS into a hyphenated method (GC-MS) for chemical identification provides orthogonality, and thus definitive analytical information for many types of samples including chemical unknowns within mixtures, with a high degree of certainty [1,14]. Effective analysis of chemical compounds has been accomplished with GC-MS systems utilizing transmission quadrupole (TQMS) and ion trap (ITMS) mass spectrometers $[15,16]$. The use of laboratory grade GC-MS systems for field applications is constrained due to the lack of ease of transportability. Numerous advancements have been made in the development of deployable GC-MS systems. Nevertheless, the necessity and the ability to transport, transfer, and effectively use analytical equipment in the field has been challenging due to the limitations of size, speed, power, and ruggedness.

There are numerous analytical instruments currently being used by military, and other non-traditional workers, for chemical identification, including a number of portable GC instruments which have been shown to be well-suited for the identification and quantification of expected analytes. Field-portable (FP) GC-MS systems have been developed and applied in many settings including forensic investigations of explosives, fires, airborne toxicants in war zones, natural disasters, and environmental assessments $[1,12,17]$. Nevertheless, the early systems suffered from incapability to perform liquid injections, poor chromatography related to the GC-MS membrane interfaces, inability to analyze compounds with relatively low volatility, inadequate battery life, slow analysis, and high maintenance requirements $[1,13]$. As a result of increased market demand for truly field-portable systems, considerable effort has gone into improving capabilities of field-portable GC-MS instruments, including portability and performance [4]. Well-known advances include the use of low thermal mass (LTM) resistively heated GC capillary column assemblies which significantly increase sample throughput and speed while simultaneously reducing the overall instrument size $[2,18]$.

The small LTM GC assembly is capable of rapid analysis with high chromatographic performance, and when combined with small MS detectors, further instrument size and power reductions are realized compared to earlier systems [2-5,7-9]. As such, a need exists for continuous improvement of FP GC-MS instrument options, and smaller systems utilizing miniature ITMS detectors have been developed to meet this need. In addition to the claim that ITMS analyzers are 10-100 times more sensitive than their ion beam quadrupole cousins, ITMS technology has allowed advances in mass analyzer miniaturization $[4,5,8,9,19]$, which has been touted as the biggest advancement for field use by military, environmental, and emergency preparedness users.

Internal ionization ITMS instruments are ideal candidates for miniaturization because they are inherently small and contain few or no ion optic elements, and thus do not require highly precise alignment. Additionally, ITMS detectors can operate at higher pressure than other MS types. This occurs because helium (He) buffer gas has an effect of collisionally dampening ion motion to 
collapse ions to the center of the ion trap [20]. Since the field is more homogenous in the center of the trap, the ions stored closer to the center of the trap will experience a more uniform ejection process, leading to improved mass spectrometer performance [20]. This ability to operate at higher pressure eases requirements for the pumping systems, thus allowing for smaller pumps that use less power $[5,20]$.

Furthermore, the amplitude of the applied radio frequency (rf) trapping potential is inversely proportional to the square of the analyzer radial dimension. Therefore, a decrease in analyzer size results in a reduction of operating voltage, hence, lower power requirements [5]. Thus, miniaturization of ITMS detectors with smaller GC systems has been explored for best potential FP GC-MS design.

Although technological advances have been made, for over a decade, chemists and other experts have reported that pseudomolecular ion formation in ITMS systems as a result of in-trap chemical reactions for certain analytes, observed in spectra as fragment/neutral adducts, protonated monomers, and dimers, are problematic for analysis by technicians and analysts in the field and may lead to chemical misidentification [9]. Though these pseudomolecular ions have been viewed as an analytical complication, it is possible that their complex spectral data can be explored for unique identification purposes.

A few manufacturers have focused on developing overall combined instrument miniaturization by coupling the smaller and faster LTM GC with miniaturized ITMS analyzers. A miniature cylindrical ion trap (CIT) mass analyzer was developed and coupled with LTM GC to form the Griffin Model 450 GC-CITMS system (ICX-Griffin, West Lafayette, IN), which has dimensions (48.8 cm x $48.8 \mathrm{~cm} \times 53.6 \mathrm{~cm}$ ) and weight ( $43.5 \mathrm{~kg}$ ) considerably smaller and lighter than laboratory or vehicle mounted transmission quadrupole GC-MS systems [5] (Figure 1a).

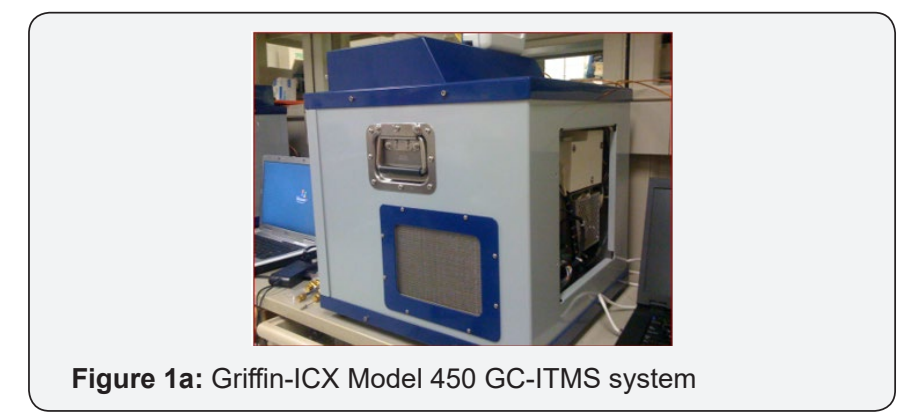

These small internal ionization ion trap GC-MS systems have the advantage of size, weight, and reduced power requirements, as well as improved sensitivity, lending to better overall portability and performance in deployed settings [1,2]. Small ITMS instruments also possess analytical capabilities not present in transmission quadrupole EI/MS detectors. The ion/molecule interactions that may be observed in internal ionization ITMS mass spectra exemplify self-chemical ionization (self-CI) leading to self-ion-molecule reactions (SIMR), and the formation of pseudomolecular ions commonly observed in mass spectra for certain analytes as fragment/neutral adducts, protonated molecules, and dimers [1,2124].

Although these SIMR reactions are often viewed as a drawback for field technicians and field analysts, self-CI in ITMS may possibly be useful for locating molecular ions in the spectra of certain classes of compounds, allowing for a definitive identification or as an additional source of evidence. Formation of characteristic peaks during self-CI can be useful for the identification of unknown compounds without the necessity of chemical reagents required in traditional $\mathrm{CI}[9,4]$. This technique could potentially be applied to perform SIMR for isomer differentiation [4-8,23]. This study focused on exploring the possibility of using FP GC- ITMS systems for isomer differentiation of hazardous and CWA-related chemicals.

\section{Experimental Methods and Procedures}

\section{Objectives}

The purpose of this study was to investigate whether SIMRs that occur in small internal ionization ITMS systems can be used to differentiate isomers of sulfides commonly seen as components or byproducts of military-relevant chemicals, such as CWAs.

\section{Materials and Methods}

The Griffin Model 450 (Figure 1a) FP GC-ITMS system (Griffin-ICX Technologies, West Lafayette, IN) was used to analyze the chemicals of interest. The Griffin FP GC-ITMS is outfitted with a low thermal mass (LTM) Gas Chromatograph. For this study, it was equipped with a $30 \mathrm{~m}$ resistively heated DB-5MS capillary GC column. Ultra-high purity (UHP) helium was used as a carrier gas for every experiment. The injector temperature was set at $250^{\circ} \mathrm{C}$, and the initial column temperature was set to $40^{\circ} \mathrm{C}$ with a temperature hold time of 60 s.

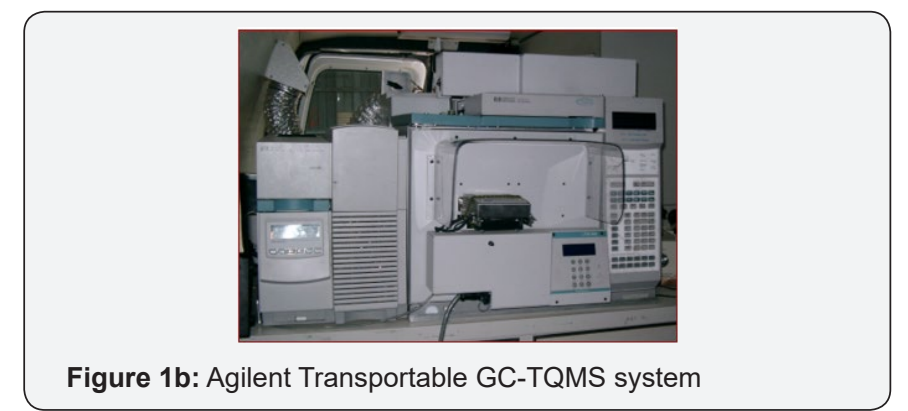

The temperature ramp rate was established at $40^{\circ} \mathrm{C}$ per minute to a terminal column temperature of $300^{\circ} \mathrm{C}$, and terminal temperature hold time of $60 \mathrm{~s}$. The instrument was operated in splitless mode, and the mass range was 40 to $425 \mathrm{amu}$. The FP GC-ITMS was used to obtain mass spectra for propyl and butyl sulfide isomers. In this study, dipropyl, isopropyl, di-n-butyl, and sec-butyl sulfides were analyzed. The sulfides were obtained commercially (Aldrich, Milwaukee, WI). Spectral analysis for the ITMS instrument was conducted using the manufacturer-designed Griffin Systems Software (GSS) program (Griffin-ICX Technologies, West Lafayette, IN). 
The experiment was repeated using a laboratory-grade Agilent 6890 GC coupled with a 5975 TQMS (Agilent Technologies, Wilmington, DE). Sulfide sample introduction was performed using solid phase microextraction for both systems. For both instruments, the injector temperature, initial column (Figure 1b) temperature, initial temperature hold, temperature ramp rate, terminal column temperature, and terminal temperature hold were identical, and both were operated under split less injection mode.

The small ITMS instrument was compared with the TQMS for the ability to differentiate sulfide isomers. The mass spectra produced during the analysis of the introduced sulfide isomers were examined for formation of pseudomolecular ions as protonated molecules $[\mathrm{M}+\mathrm{H}]+$, adducts $[\mathrm{M}+\mathrm{F}]+$, and dimers $[2 \mathrm{M}+\mathrm{H}]+$. Data collected using TQMS was analyzed with the Agilent MSD ChemStation software (Agilent Technologies, Wilmington, DE). The National Institute of Standards and Technology Mass Spectral Library (NIST, Gaithersburg, MD) was used as a reference standard for spectral analysis.

\section{Results}

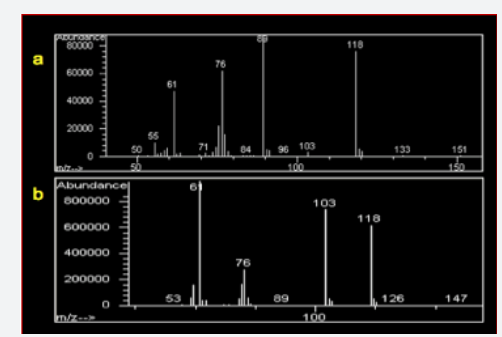

Figure $2(\mathbf{a}, \mathbf{b})$ : TQMS spectra of (a) dipropyl and (b) isopropyl sulfides, both appearing similar and exhibiting the 118 molecular ion, as expected.

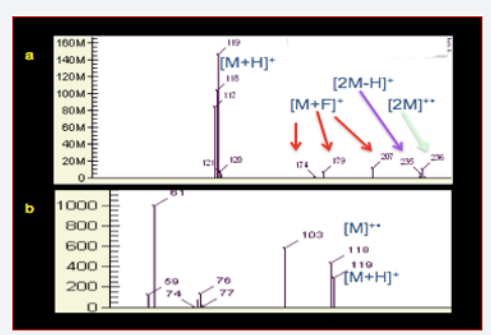

Figure $3(\mathbf{a}, \mathbf{b})$ : ITMS spectra of (a) dipropyl and (b) isopropyl sulfides, both exhibiting different pseudomolecular ions observed.

Isomers of propyl and butyl sulfides were analyzed using laboratory grade and FP GC-MS systems with TQMS and ITMS detectors, respectively. Dipropyl and isopropyl sulfides were also analyzed using both instruments. As exhibited in Figures 2a,b, there is little difference between the isomers when analyzed with TQMS. The molecular ion $[\mathrm{M}+]$ was observed at $\mathrm{m} / \mathrm{z} 118$, as expected for both isomers. When analyzed with the ITMS, pseudomolecular ions were observed for both isomers (Figure 3).

The formation of $[\mathrm{M}+\mathrm{H}]+$ at $\mathrm{m} / \mathrm{z} 119,[\mathrm{M}+\mathrm{F}]+$ at $\mathrm{m} / \mathrm{z} 174,179$, and 207 , and the dimer ions $[2 \mathrm{M}]+$ and $[2 \mathrm{M}-\mathrm{H}]+$ at $\mathrm{m} / \mathrm{z} 235$ and 236 respectively, were observed for propyl sulfide (Figure 3a).
Only the molecular ion $(\mathrm{m} / \mathrm{z} 118)$ and $[\mathrm{M}+\mathrm{H}]+(\mathrm{m} / \mathrm{z} 119)$ were observed for isopropyl sulfide (Figure $3 \mathrm{~b}$ ). The results from the analyses of the butyl sulfides were similar. TQMS analysis for the butyl sulfide isomers were similar and no pseudomolecular ions were observed (Figure 4).

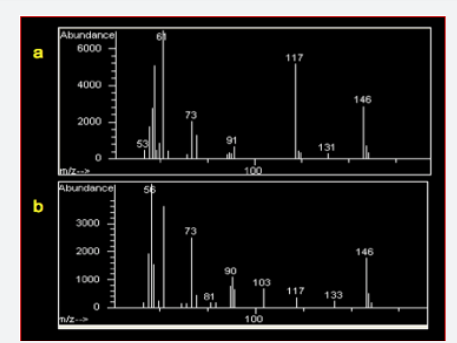

Figure 4: TQMS spectra of (a) di-n-butyl and (b) sec-butyl sulfides, both appearing similar and exhibiting the 146 molecular ion, as expected.

The molecular ion for both was observed at m/z 146 as expected. ITMS analysis yielded the formation of pseudomolecular ions for both isomers. For di-n-butyl sulfide, $[\mathrm{M}+\mathrm{H}]+$ was observed at $\mathrm{m} / \mathrm{z} 147$, as exhibited in Figure 5a. Adducts were also observed $(\mathrm{m} / \mathrm{z} 202,207$, and 249) as well as dimer ions [2M+H]+ and [2M]+ (m/z 292 and 293, respectively). The protonated monomers and adducts $[\mathrm{M}+\mathrm{H}]+(\mathrm{m} / \mathrm{z} 147)$ and $[\mathrm{M}+\mathrm{F}]+(\mathrm{m} / \mathrm{z} 201,207)$ were observed when sec-butyl sulfide was analyzed with the ITMS, but no dimer was observed (Figure 5b).

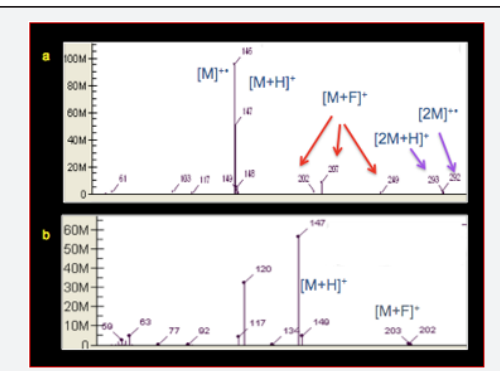

Figure 5(a,b): ITMS spectra of (a) di-n-butyl and (b) sec-butyl sulfides, exhibiting different pseudo molecular ions observed.

Table 1: Characteristics of ITMS spectra observed for sulfide isomer analytes. Protonated molecules, dimers, and adduct ions are not observed using electron ionization with a TQMS detector.

\begin{tabular}{|c|c|c|c|}
\hline Analyte & {$[\mathbf{M + H}]^{+}$} & Dimer(s) & Adduct(s) \\
\hline dipropyl sulfide & Yes & Yes $^{1,2}$ & Yes \\
\hline diisopropyl sulfide & Yes & No & No \\
\hline di-n-butyl sulfide & Yes & Yes $^{2,3}$ & Yes \\
\hline sec-butyl sulfide & Yes & No & Yes \\
\hline
\end{tabular}

Due to low pressure and rapid transit of ions created in the ion beam TQMS instrument, ion/molecule reactions do not occur, and resulting mass spectra are consistent and standardized. In contrast, the ITMS instrument produced ion current for the pseudomolecular ions for all four analytes as predicted. The differentiation results can be seen in Figures 2-5 and Table 1. The resulting presence/absence of protonated monomers, adducts, and dimers 
were used to differentiate the sulfide isomers.

\section{Discussion}

Formation of pseudomolecular ions as protonated molecules, adducts, and dimers may be affected by steric effects in which the size, space, or arrangement of atoms within the molecule affect the energies and potential reactions. Additionally, the sizes and geometries of side chains in the studied analytes can have an effect on electrophilicity of cations produced by EI, and thus the ability to react. A branched structure will tend to have more resonance structures when compared to a straight chain, leading to electrophile stabilization. Thus, branching tends to result in a decrease of reactions and a subsequent reduction or elimination of adducts observed.

This phenomenon was observed during the analysis of the propyl sulfide and butyl sulfide isomers. The straight-chain isomers (propyl and dibutyl sulfide) both exhibited the formation of $[\mathrm{M}+\mathrm{H}]+$, multiple adducts, and multiple dimers, compared to the branched chain isomers, which exhibited fewer pseudomolecular ions. These resulting differences in adducts and dimers observed in mass spectra, between isomers, can be used to differentiate those isomers from one another, potentially allowing for a higher degree of certainty during routine or emergency response chemical identification operations.

The resulting presence/absence of protonated monomers, adducts, and dimers were used to differentiate the sulfide isomers, showing an advanced capability not possible with larger transportable systems with TQMS detectors. Therefore, although the pseudomolecular ion formation that occurs as a result of reactions in the ion trap may make identification slightly difficult for an inexperienced or untrained technician or analyst, increased and improved education and training may not only correct this ability gap, but may improve the overall capability of field analysts to identify compounds with a higher degree of certainty as a result of the capability to differentiate isomers.

\section{Other Considerations}

\section{Use of GC-ITMS technologies in combination with mod- ern and emerging computer-based communications technologies}

Additionally, despite the technical and scientific gaps that potentially exist between lab scientists and field technicians and analysts, the gap may also potentially be closed as a result of emerging technologies in high-speed internet, bandwidth, cloud computing, cyber-security, and telepresence communications. Miniaturization, speed, capability, and power of analytical instruments has been critical to field-based analysis and near real-time answers regarding potential exposures. However, advancements in other technological arenas are also potentially beneficial to emergency preparedness and response, among other field-based operations.

Numerous advancements have been made in the world of wireless high-speed internet, bandwidth availability, and cloud computing. There is the potential that gaps in technician training, ability, and knowledge can be overcome if data collected by technicians in the field can be transported and analyzed in real-time by subject matter expert scientist analysts located at a distance. This can be potentially accomplished through the use of existing video communication technology platforms in combination with secure E-mail and other streaming technologies, as well as through the use of emerging communication technologies such as holoportation, an advanced system that uses mixed reality and heads up displays to provide near telepresence.

\section{Use of GC-ITMS technology with combination of holo- portation communications}

Mixed reality technologies combine virtual reality and augmented reality (VR/AR), and are being studied for and employed in medicine, education, psychological treatment, architecture, rehabilitation, and tourism [25,26-31], among other fields. The advancement of VR/AR devices has increased the way communications can be used. Mixed reality involves using computer hardware, software, capture devices, displays, sensor, and trackers for the effective collection, compression, and dissemination of the aggregated data [29].

What this does is allow users to insert virtual content into real world content, permitting both to run simultaneously in real-time [26]. The technology has already been used repeatedly for inter-group military participation, including multi-participant battlefield exercises involving multiple defense agencies, in real-time. But even these uses fall short compared to the emerging capability of telepresence, which is the ability of a participant to appear present at a remote location. When combined, VR/AR allow scenarios that are nearing complete telepresence through what is called holoportation [31,32].

Holoportation takes not only distance communication, but VR/AR to another level, combining these modalities to produce technology that offers a dynamic, 3-D holographic presence. This technology is practically an end-to-end system of real-time reconstructions of space, surroundings, and people, with the ability to transmit those recreations to remote users, affording immediate, 3 -D visual, auditory, and emotional interactions between users at remote settings [33].

In this technological method, a series of specially outfitted cameras is situated in the space to capture the participants and their surroundings three-dimensionally. These cameras capture the data and the developed software fuses the data to create a temporally consistent model. These cameras are also outfitted with devices such as the Hololens ${ }^{\mathrm{TM}}$ tracking technology that aid the cameras in taking the 3-D models and compositing them in real-time into the real world. The reconstructions are textured, and the complete data are compressed and transmitted to similar set-ups on the other end, where the receiving user wearing the Hololens $^{\mathrm{TM}}$ can see the remote participant live, in his or her space, as though they are co-present in the same location $[33,34]$.

Holoportation allows people who are distances away to have 
the experience of being present in the same time and space, offering numerous advantages. Holoportation can potentially connect technicians in the field with scientists and other subject matter experts in the lab or other safe location, even thousands of miles away, and allow the experts to perform observation and analysis of the chemical data sampled using the chemical analyzers, providing an expert and more definitive answer from miles or thousands of miles away for operational decision-makers in the danger-zone [31]. Thus, whether it be performed through currently available 2-D platforms in combination with E-mail transmission of data files, or through the emerging telepresence available through holoportation, it appears that there is a potential that the challenge of chemical analysis and the complexities of spectral differentiation by complex systems may fall not into the hands and minds of field technicians and scientists, but perhaps in the hands of cyber-security and cryptology professionals that must ensure data integrity and security.

\section{Conclusion}

Exposure scientists use GC-MS systems for analysis of hazardous chemicals and materials to prevent military exposures, environmental exposures, public health issues, and to gather forensic evidence. Because even the most transportable GC-MS systems with TQMS detectors historically have limited portability due to size, power, and ruggedness concerns, small ITMS have been manufactured to reduce size and increase ruggedness [2,4-8] of combined GC-MS systems, making them more apt for deployment to the field by military special operations, environmental health, and CBRNE experts.

Despite the benefits of significantly smaller instruments due to the ability to miniaturize GC-ITMS instruments, the complexities of analysis of ITMS spectra, compared to its TQMS cousin, have been touted as a potential hindrance to operational effectiveness, particularly due to the formation of pseudomolecular ions observed in ITMS spectra. Some analysts and scientific strategists believe that more complex spectra potentially leading to chemical misidentification by operators, technicians, and field analysts, generally requiring a more experienced GC-MS subject matter expert to ensure accuracy of data $[9,4]$.

However, the chemical reactions that occur in the ion trap and lead to the formation of the pseudomolecular ions may not be as much of a drawback as some believe. It was proposed in this study that these spectra can actually improve analytical power if the pseudomolecular ions are used to differentiate chemical isomers, not possible with TQMS. The ability to not only identifying the chemicals but being able to actually differentiate isomers leads to an increased level of identification as well as an additional level of identity confirmation. The researchers analyzed isomers of propyl and butyl sulfide using GC-TQMS and GC-ITMS instruments and compared the spectral analyses. TQMS spectra exhibited nearly identical peaks for sulfide isomers. ITMS yielded complex spectra with protonated molecules, dimers, and adducts. Differentiation of the sulfide isomers were accomplished via characteristic spectral differences in the pseudomolecular ions formed in the ion trap.

In summary, field-portable FP GC-MS systems with miniature ITMS detectors can be used to differentiate chemical isomers through analysis of their self-CI byproducts, a capability that can be important for analysis of toxic industrial chemicals, chemical warfare agents, and other compounds important to military, emergency response, and forensic professionals, among others. Thus, the self-CI and the formation of pseudomolecular ions previously viewed as a drawback can actually be used as an attribute of the smaller detectors. Furthermore, in a time of rapidly advancing technological emergence, the gaps and weaknesses supposed to be deficiencies of the use of these systems in field settings may be void as analytical assessments, processes, and determinations can be made by remote analysts that are potentially able to analyze transmitted data in near real-time, or even evaluate the data and instruct technicians through the use of telepresence technologies, such as holoportation. Future research should explore quantification of chemical compounds using pseudomolecular ion data. Additional research should be conducted regarding the collection, transmission, distance-analysis, and telepresent analysis of chemical compounds.

\section{Disclaimer Statement}

This project was not funded or sponsored by any particular institution. It consists of independent research by the authors, and the authors alone are responsible for the findings and opinions of the research as presented in the article. Although the authors were affiliated with numerous institutions during the course of the research and manuscript development, the findings, interpretations, opinions, and statements discussed in the article do not represent any official stance or position of Changineering Global, Nova Southeastern University, The Uniformed Services University of the Health Sciences, Naval Hospital Camp Pendleton, The Academy of Interdisciplinary Health Science Leaders, The Defense Threat Reduction Agency, the US Navy, or the US Army.

\section{References}

1. Eckenrode BA (2001) Environmental and forensic applications of fieldportable GC-MS: An overview. Journal of the American Society for Mass Spectrometry 12(6): 683-693.

2. Smith PA, Sng MT, Eckenrode BA, Leow SY, Koch D, et al. (2005) Towards smaller and faster gas chromatography-mass spectrometry systems for field chemical detection. J Chromatogr A 1067(1-2): 285-294.

3. Burroughs GE, Woodfin WJ (1995) On-site Screening for Benzene in Complex Environments. American Industrial Hygiene Association Journal 56: 874-882.

4. Lee ED, Smith PA, Bowerbank CR, Later DW (2010) Rapid Chemical Threat Identification by SPME-GC-TMS. In: Spectroscopy: Solutions for Materials Analysis.

5. Patterson GE, Guymon AJ, Riter LS, Everly M, Griep-Raming J, et al. (2002) Miniature cylindrical ion trap mass spectrometer. Analytical Chemistry 74(24): 6145-6153.

6. Lammert SA, Rockwood AA, Wang M, Lee ML, Lee ED, et al. (2006) Miniature toroidal radio frequency ion trap mass analyzer. J Am Soc Mass Spectrom 17(7): 916-922. 
7. Welsh MS, Lamesse M, Karpinski E (2000) The verification of hazardous ingredients disclosures in selected material safety data sheets. Applied Occupational and Environmental Hygiene 15(5): 409-20.

8. Contreras JA, Murray JA, Tolley SE, Oliphant JL, Tolley HD, et al (2008) Hand-Portable Gas Chromatography-Toroidal Ion Trap Mass Spectrometer (GC-TMS) for Detection of Hazardous Compounds Journal of the American Society for Mass Spectrometry 19(10): 14251434.

9. Smith PA, Lepage CJ, Lukacs M, Martin N, Shufutinsky A, et al. (2010) Field-portable gas chromatography with transmission quadrupole and cylindrical ion trap mass spectrometric detection: Chromatographic retention index data and ion/molecule interactions for chemical warfare agent identification. International Journal of Mass Spectrometry 295(3): 113-118.

10. Talbot TB, Lukey B, Platoff GE (2008) Introduction to the Chemical Threat. In: Lenhart MK, editor. Medical Aspects of Chemical Warfare. Borden Institute and Office of the Surgeon General United States Army: Washington, DC, USA.

11. Mc Glown KJ (2004) Terrorism and Disaster Management: Preparing Healthcare Leaders for the New Reality. Health Administration Press: Chicago, USA.

12. Smith PA, Kluchinsky TA, Savage PB, Erickson RP, Lee AP, et al. (2002) Traditional Sampling with Laboratory Analysis and Solid Phase Microextraction Sampling with Field Gas Chromatography/Mass Spectrometry by Military Industrial Hygienists. AIHA Journal, 63(3): 284-292.

13. Smith PA, Lepage CRJ, Koch D, Wyatt HDM, Hook GL, et al. (2004) Detection of gas-phase chemical warfare agents using field-portable gas chromatography-mass spectrometry systems: instrument and sampling strategy considerations. Trends Anal Chem 23(4): 296-306.

14. Gross JH (2004) Mass Spectrometry: A Textbook. Springer: Berlin, Germany.

15. Ono-Ogasawara M, Myojo T, Smith TJ (2008) A simple direct injection method for GC/MS analysis of PAHs in paticulate matter. Industrial Health 46(6): 582-593.

16. Joos PE, Godoi AFL, DeJong R, deZeeuw J, Grieken RV (2003) Trace analysis of benzene, toluene, ehtylbenzene and xylene isomers in environmental samples by low-pressure gas chromatography-ion trap mass spectrometry. J Chromatogr A 985(1-2): 191-96.

17. McMahon G (2007) Analytical Instrumentation: A Guide to Laboratory, Portable and Miniaturized Instruments.: Wiley \& Sons: West Sussex, England.

18. Sloan KM, Mustacich RV, Eckenrode BA (2001) Development and evaluation of a low thermal mass gas chromatograph for rapid forensic GC-MS analyses. Field Analytical Chemistry Technol 5: 288-301.

19. Riter LS, Peng Y, Noll RJ, Patterson GE, Aggerholm T, et al. (2002) Analytical performance of a miniature cylindrical ion trap mass spectrometer. Analytical Chemistry 74(24): 6154-6162.

20. Stafford G (2002) Ion trap mass spectrometry: A personal perspective. Journal of the American Society for Mass Spectrometry 13(6): 589-596.
21. Wu HF, Ho MY (2001) Selective self-ion/molecule reactions in both external and internal ion trap mass spectrometers. Rapid Communication in Mass Spectrometry 15(5): 1309-1316.

22. Wu HF, Chen CH, Wu MT (2004) Observation of self-ion-molecule reactions during collisionally activated dissociation in an ion-trap mass spectrometer. Journal of Mass Spectrometry 39(4): 396-401.

23. Wu HF, Chuan YJ (2003) Isomer differentiation by combining gas chromatography, selective self-ion/molecule reactions and tandem mass spectrometry in an ion trap mass spectrometer. Rapid Communications in Mass Spectrometry 17(10): 1030-1036.

24. Wu HF, Wu WF (2003) Comparing differentiation of xylene isomers by electonic ionization, chemical ionization and self-ion/molecule reactions and the first observaton of methyne addition ions for xylene isomers in self-ion/molecule reactions for non-nitrogenated compounds. Rapid Communication in Mass Spectrometry 17: 23992406.

25. Abdelfatah H (2016) Cyber Learning from Reality through Virtuality to Holoportation: Status-Quo and a Proposed Model for Closing the Gap in Africa. ASRO Journal of Education 1(2): 28-38.

26. Chicchi-Giglioli IA, Pallavicini F, Pedroli E, Serino S, Riva G (2015) Augmented reality: A Brand-New Challenge for the Assessment and Treatment of Psychological Disorders. Computational and Mathematical Methods in Medicine.

27. Jenny S (2017) Enhancing tourism with augmented and virtual reality. HAMK: Visamaki, Finland.

28. Khor WS, Baker B, Amin K, Chan A, Patel K, et al. (2016) Augmented and virtual reality insurgery-the digital surgical environment: applications, limitations and legal pitfalls. Annals of translational medicine 4(23): 454.

29. Kim Y, Kim H, Kim YO (2017) Virtual reality and augmented reality in plastic surgery: A review. Archives of plastic surgery 44(3): 179-187.

30. Moro C, Štromberga Z, Raikos A, Stirling A (2017) The effectiveness of virtual and augmented reality in health sciences and medical anatomy. Anatomical Sciences Education 10(6): 549-559.

31. Shufutinsky A, Sibel J, Murray-Hayden P, Shufutinsky D, Cox R (2018) Holorportation: The Disruptive Technology that Will Change the Practice of Conflict Analysis and Resolution. OD PRACTITIONER 50(3): 56-63.

32. Orlosky J, Kiyokawa K, Takemura H (2017) Virtual and augmented reality on the 5G highway. Journal of Information Processing 25: 133141.

33. Orts-Escolano S, Rhemann C, Fanello S, Chang W, Kowdle A, et al. (2016) Holoportation: Virtual 3d teleportation in real-time. In Proceedings of the $29^{\text {th }}$ Annual Symposium on User Interface Software and Technology.

34. Lounsbury D (2003) Military Preventive Medicine. In: Kelley P (Ed.), Military Preventive Medicine: Mobilization and Deployment (Vol. 1, pp. XVI), Washington DC: Borden Institute, USA. 


\section{Your next submission with Juniper Publishers will reach you the below assets}

- Quality Editorial service

- Swift Peer Review

- Reprints availability

- E-prints Service

- Manuscript Podcast for convenient understanding

- Global attainment for your research

- Manuscript accessibility in different formats

( Pdf, E-pub, Full Text, Audio)

- Unceasing customer service

Track the below URL for one-step submission https://juniperpublishers.com/online-submission.php 